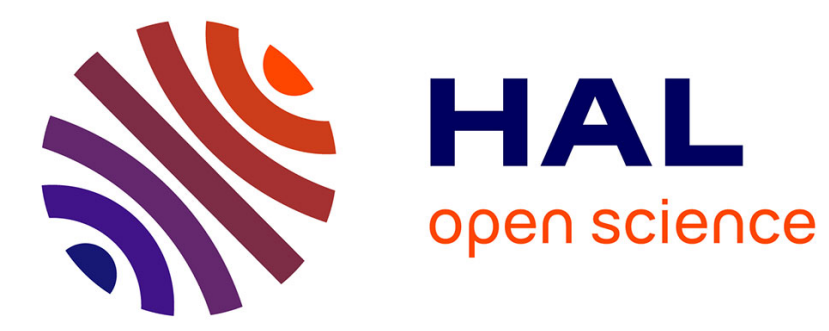

\title{
Gas flow with low gas pressure in porous media. Quasi-statics and acoustics
}

\author{
Juliette Chastanet, Pascale Royer, Jean-Louis Auriault
}

\section{To cite this version:}

Juliette Chastanet, Pascale Royer, Jean-Louis Auriault. Gas flow with low gas pressure in porous media. Quasi-statics and acoustics. 2nd Biot conference on poromechanics, Aug 2002, Grenoble, France. hal-01718797

\author{
HAL Id: hal-01718797 \\ https://hal.science/hal-01718797
}

Submitted on 8 Aug 2018

HAL is a multi-disciplinary open access archive for the deposit and dissemination of scientific research documents, whether they are published or not. The documents may come from teaching and research institutions in France or abroad, or from public or private research centers.
L'archive ouverte pluridisciplinaire HAL, est destinée au dépôt et à la diffusion de documents scientifiques de niveau recherche, publiés ou non, émanant des établissements d'enseignement et de recherche français ou étrangers, des laboratoires publics ou privés. 


\title{
Gas flow with low gas pressure in porous media: quasi-statics and acoustics
}

\author{
J. Chastanet, P. Royer \& J.L. Auriault \\ Lab. 3S, UJF, INPG, CNRS, Domaine Universilaire, France
}

ABSTRACT: This work deals with the mathematical modelling of gas flow with low gas pressure in porous media. At the pore scale, this type of flow is characterized by a wall-slip effect which at the sample scale entails a dependence of permeability upon the pressure. This latter property is described by Klinkenberg's law. The goal of the present work is to examine the robustness of this law. The mathematical modelling is performed using an upscaling technique, namely the homogenisation method for multiple scales. Two distinct problems are investigated: i) the flow of gas at low pressure in a composite porous media; ii) acoustics with wall-slip effect of a porous medium saturated by a gas. In both cases, it is shown that, in the most general cases, Klinkenherg's law is not verified at the macroscopic scale.

\section{INTRODUCTION}

In general cases, gas flow in porous media is described by Darcy's law: $v=-k_{\infty} \nabla_{p}$, in which the permeability $k_{\infty}$ is an intrinsic property of the medium. At low gas pressure, the observed gas filtration velocity is greater than that predicted by Darcy's law. This is due to the large value of the mean free path of gas molecules, which is inversely proportional to the pressure. In effect, a large mean free path gives rise, at the pore scale, to a wall-slip on the solid/fluid interface. The no-slip condition which is implicitly assumed when considering Darcy's law is therefore no longer valid. For describing such flows, Klinkenberg (1941) introduced the concept of gas permeability:

$k_{g}=k_{\infty}\left(1+\frac{4 c \lambda}{r}\right)=k_{\infty}\left(1+\frac{b}{\bar{p}}\right)$

in which $c$ is a constant, $\lambda$ is the mean free path, $\bar{p}$ is the average pressure over the sample, $r$ is the pore size and $b$ is a $p$-independent parameter. This effect may take place even at atmospheric pressure if the pore-size is sufficiently small. The determination of parameter $b$ has been the subject of a few experimental (Bachr \& Hult 1991; Jones 1972) numerical (Fun-GauHo \& Strieder 1982) and theoretical works (Wu et al. 1998; Skjetne \& Gudmundsson 1993). This parameter $b$ may take a large range of values from 0.1 for media of great permeability (Klinkenberg 1941) to 18 for low permeability media (Wu et al. 1998). In Skjetne \& Auriault (1999), Klinkenberg's law is rigorously derived by homogenisation; the fluid flow equations and the wall-slip condition are considered at the porescale. Homogenisation of this local description leads to the macroscopic model which includes a tensorial form of $(1)$ in which the term $1 / \bar{p}$ is replaced by $1 / p$, where $p$ is the pressure field. The gas permeability is therefore independent of the boundary conditions.

The aim of the present work is to examine whether the strueture of Klinkenberg's law does survive the upscaling procedure. Two distinct problems are investigated: i) the flow of gas at low pressure in a composite porous media; ii) acoustics with wall-slip effect of a porous medium saturated by a gas. The up-scaling technique is the homogenisation method of multiple scale expansions introduced by Benssoussan et al. (1978) and Sanchez-Palencia (1980). In section 2, we consider the flow of gas at low pressure in a porous composite. The local scale under consideration is the composite scale: it consists of two interconnected constituents in which Klinkenberg's law is supposed to be valid. This local description is homogenised. We show that the $1 / p$-form of Klinkenberg's law is not always verified: it depends upon the medium geometry. In section 3, we investigate the acoustics of wall-slip gas flow in porous media. For this problem, the local scale under consideration is the pore scale . and the local description consists of the equations of linear acoustics with a wall-slip condition over the solid-liquid interface (Skjetne \& Auriault 1999). The macroscopic description derived by homogenisation reveals the fragility of Klinkenberg's law. Memory effects are obtained, but which are those derived in 
the classical case of acousties in porous media (Levy 1979; Auriault 1980).

\section{GAS FLOW AT LOW PRESSURE IN A POROUS COMPOSITE}

\subsection{Medium description}

We consider a periodic composite which consists of two interconnected rigid porous constituents. Both constituents are saturated by gas at low pressure. Let denote by $\Omega$ the period of characteristic size $O(I)$. Within the period both constituents occupy domains $\Omega_{1}$ and $\Omega_{2}$ and their common interface is denoted by $\Gamma$. The porosities of both constituents are assumed to be of same order $\phi_{1}=O\left(\phi_{2}\right)$. The whole structure is subject to a macroscopic pressure gradient of characteristic length $L$. Assuming that Klinkenberg's law is valid in both constituents, over the period the isothermal flow of gas at low pressure is described by the following equations:

$\phi_{u} \frac{\partial p_{\alpha}}{\partial t}-\nabla \cdot\left(p_{u} K_{u} \nabla p_{u}\right)=0 \quad$ in $\Omega_{u}$,

$[p K \nabla p]_{\Gamma} \cdot \boldsymbol{n}=0 \quad$ on $\Gamma$,

$p_{1}=p_{2}$ on $\mathrm{\Gamma}$,

in which

$K_{\alpha}=k_{\alpha}\left(1+\frac{b_{u}}{p_{\alpha}}\right)>0 \quad(\alpha=1,2)$.

and where $p_{\alpha}$ represents the gas pressure in $\Omega_{\alpha}, k_{\alpha}$ denotes the intrinsic permeability of $\Omega_{\alpha}, \phi_{\alpha}$ is the porosity, $A$ is a constant, $\boldsymbol{n}$ is a unit vector normal to $\Gamma$ and $[\varphi]_{\Gamma}$ denotes the discontinuity of $\varphi$ over $\Gamma$. Constants $b_{1}$ and $b_{2}$ depend upon the pore geometries.

Let now apply the homogenisation procedure with the approach suggested by Auriault (1991). The fundamental assumption behind homogenisation is that the scales must be separated: $l<<L$ and the key parameter of the method is $\varepsilon=l / L$. The first step consists in normalizing the local description and the non- dimensional numbers must be estimated with respect to powers of 8 . Considering $/$ as the reference characteristic length and assuming that both permeabilities are of same order, two non-dimensional numbers remain to be estimated:

$Q_{\alpha}=\frac{\left|\phi_{\alpha} \frac{\partial p_{\alpha}}{\partial t}\right|}{\left|\nabla \cdot\left(p_{\alpha} K_{\alpha} \nabla p_{\alpha}\right)\right|} \quad(\alpha=1,2)$.

Both parameters $Q_{1}$ and $Q_{2}$ characterize the transient behaviour of the flow in both consituents and are of same order: $Q_{1}=O\left(Q_{1}\right)=O\left(Q_{2}\right)$. It can be shown (Auriault 1983) that a macroscopic transient regime, which is the case of greatest interest, corresponds to: $Q_{1}=O\left(\varepsilon^{2}\right)$. Therefore, the dimensionless local description is written as follows:

$\varepsilon^{2} \phi_{\alpha} \frac{\partial p_{\alpha}}{\partial t}-\nabla \cdot\left(p_{\alpha} K_{\alpha} \nabla p_{\alpha}\right)=0$ in $\Omega_{\alpha}$,

$[p K \nabla p]_{\Gamma} \cdot \boldsymbol{n}=0$ on $\Gamma$,

$p_{1}=p_{2}$ on $\Gamma$.

For simplicity we have kept the same notations, but all quantities in equations (7) (9) are now dimensionless quantities.

\subsection{Homogenisation}

As a result of the separation of scales, two dimensionless space variables may be defined: $\boldsymbol{y}-\boldsymbol{X} / \mathbf{l}$ and $\boldsymbol{x}=\boldsymbol{X} / L$, where $\boldsymbol{X}$ is the physical space variable. Since $l$ has been chosen as the reference characteristic length, the dimensionless gradient operator is therefore $\nabla_{y}+\varepsilon \nabla_{x}$, where $\nabla_{y}$ and $\nabla_{x}$ are the gradient operators with respect to variables $\boldsymbol{y}$ and $\boldsymbol{x}$, respectively. The homogenisation method is based upon the fundamental statement that if the scales are well separated, then all physical variables can be looked for in the form of asymptotic expansions in powers of $\varepsilon$ :

$\varphi=\varphi^{(0)}(\boldsymbol{y}, \boldsymbol{x})+\epsilon \varphi^{(1)}(\boldsymbol{y}, \boldsymbol{x})+\varepsilon^{2} \varphi^{(2)}(\boldsymbol{y}, \boldsymbol{x})+\ldots$

in which the functions $\varphi^{\prime}$ are $y$-periodic. Thus, the method consists in incorporating these asymptotic expansions in the dimensionless local description (7)-(9). Then, solving the boundary-value problems arising at the successive orders of $\varepsilon$ leads to the macroscopic description.

\subsubsection{First-onder pressure fields}

The first-order boundary-value problem reads

$\nabla_{y} \cdot\left(p_{\alpha}^{(0)} K_{\alpha}^{(0)} \nabla_{y} p_{\alpha}^{(0)}\right)=0 \quad$ in $\Omega_{\alpha}$,

$\left[p^{(0)} K^{(0)} \nabla_{y} p^{(10)}\right]_{\mathrm{r}^{\prime} \cdot \boldsymbol{n}=0}$ on $\mathrm{\Gamma}$,

$p_{1}^{(0)}=p_{2}^{(0)}$ on $\Gamma$,

in which $p_{\alpha}^{(0)}$ and $K_{\sigma}^{(0)}$ are $y$-periodic and where

$K_{\alpha}^{(0)}=k_{\alpha}\left(1+\frac{b_{\alpha}}{p_{\alpha}^{(0)}}\right)$.

This is a well-posed problem of unknowns $p_{a}^{(0)}$ and $K_{u}^{(0)}$. As a result of the positivity of $p_{u}^{(0)}$, we get:

$p_{1}^{(0)}=p_{2}^{(0)}=p^{(0)}(\boldsymbol{x}, t)$,

$K_{1}^{(0)}=K_{1}^{(0)}(x, t)$ and $K_{2}^{(0)}=K_{2}^{(0)}(x, t)$. 


\subsubsection{Second-order pressure fields}

At the next order, the set (7)-(9) gives the following boundary value problem of unknown $p_{\alpha}^{(1)}$ :

$\nabla_{y} \cdot\left(K_{\alpha}^{(0)}\left(\nabla_{y} p_{\alpha}^{(1)}+\nabla_{x} p^{(0)}\right)\right)=0 \quad$ in $\Omega_{\alpha}$,

$\left[K^{(0)}\left(\nabla_{y} p^{(1)}+\nabla_{x} p^{(0)}\right)\right]_{\Gamma} \cdot \boldsymbol{n}=0 \quad$ on $\Gamma$,

$p_{1}^{(1)}=p_{2}^{(1)}$ on $\Gamma$,

in which $p_{\alpha}^{(1)} y$-periodic. It can be shown that the solution is written as:

$p^{(1)}=\boldsymbol{\tau}\left(\boldsymbol{y}, p^{(0)}\right) \cdot \nabla_{x} p^{(0)}+\vec{p}^{(1)}(\boldsymbol{x}, t)$,

where $\bar{p}^{1}(\boldsymbol{x}, t)$ is an arbitrary function. Vector $\boldsymbol{\tau}$ is the solution to the well-posed local problem, which is obtained by introducing expression (20) into (17)-(19).

\subsubsection{Derivation of the macroscopic description}

At the third order, equation (7) in $\Omega_{\alpha}$ reads:

$$
\begin{aligned}
\phi_{a} \frac{\partial p^{(0)}}{\partial t}-\nabla_{x} \cdot\left(p^{(0)} K_{\alpha}^{(0)}\left(\nabla_{y} p_{\alpha}^{(1)}+\nabla_{x} p^{(0)}\right)\right) \\
-\nabla_{y} \cdot\left[p^{(0)} K_{\alpha}^{(0)}\left(\nabla_{y} p_{\alpha}^{(2)}+\nabla_{x} p_{\alpha}^{(1)}\right)\right] \\
-\nabla_{y} \cdot\left[\left(p^{(0)} K_{\alpha}^{(1)}+p_{\alpha}^{(1)} K^{(0)}\right)\right. \\
\left.\times\left(\nabla_{y} p_{\alpha}^{(1)}+\nabla_{x} p^{(0)}\right)\right]=0 .
\end{aligned}
$$

Integrating equation (21) over $\Omega_{1}$ and $\Omega_{2}$ and then using the divergence theorem, the condition of periodicity and the appropriate boundary conditions leads to the macroscopic description

$$
(\phi) \frac{\partial p^{(0)}}{\partial t}-\nabla_{x} \cdot\left(p^{(0)} \tilde{\mathcal{K}} \nabla_{x} p^{(0)}\right)=0,
$$

where $\langle\phi\rangle=\eta_{1} \phi_{1}+\eta_{2} \phi_{2}$ is the composite bulk porosity and $\eta_{\alpha}=\left|\Omega_{\alpha}\right| /|\Omega|$ is the volume fraction of constituent $\alpha$. The tensor of effective permeability $\widetilde{K}$ is defined by:

$$
K_{i j}=\frac{1}{|\Omega|} \int_{\Omega} K^{(0)}\left(I_{i j}+\frac{\partial \tau_{j}}{\partial y_{j}}\right) d \Omega
$$

It can be shown that $\tilde{\mathcal{K}}$ is symmetrical and positive. Since $\tau$ depends on $p^{(0)}$ and also upon the composite geometry, it can be seen from (23) that in the most general cases, the effective permeability doesn't verify Klinkenberg's law: depending on the geometry, the structure of expression (23) may strongly differ from the structure of (14). Therefore, Klinkenberg's law is not always prescrved after upscaling.

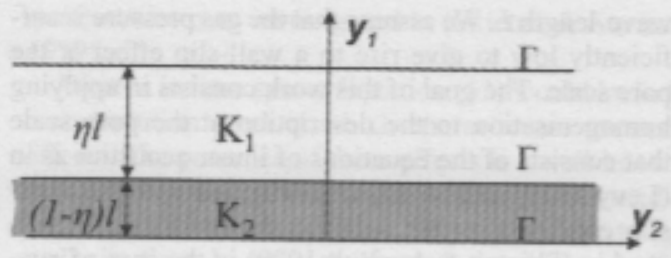

Figure 1. Bilaminated composite.

\subsection{Illustration on a simple geometry: bilaminated composite}

In order to illustrate the above results on a simple geometry, let consider a periodic bilaminated porous composite (Figure 1): it consists of two homogencous porous layers $\Omega_{1}$ and $\Omega_{2}$ of thickness $\eta l$ and $(1-\eta) l$, respectively, where $\eta$ denotes the volume fraction of layer $\Omega_{1}$. Each layer is assumed to be homogeneous and isotropic and of permeability $K_{\alpha}(\alpha=1,2)$. The effective permeability $\widetilde{\mathcal{K}}$ is given by:

$$
\begin{aligned}
& \mathcal{K}_{12}=\mathcal{K}_{13}=\mathcal{K}_{23}=0 \\
& \mathcal{K}_{22}=\mathcal{K}_{33}=\eta K_{1}+(1-\eta) K_{2}=K_{11}, \\
& \mathcal{K}_{11}=\frac{K_{1} K_{2}}{(1-\eta) K_{1}+\eta K_{2}}=K_{\perp},
\end{aligned}
$$

where $K_{||}$and $K_{\perp}$ denote the permeabilities for flows parallel and perpandicular to the layers, respectively. Introducing expression (5) for $K_{1}$ and $K_{2}$ into (25) and (26) yields

$$
\begin{aligned}
& \mathcal{K}_{22}=K_{23}=k_{22}\left(1+\frac{B_{2}}{p}\right), \\
& \mathcal{K}_{11}=\frac{k_{1} k_{2}\left(1+\frac{b_{1}}{p}\right)\left(1+\frac{b_{2}}{p}\right)}{k_{22}+\frac{k_{22} B_{2}}{p}},
\end{aligned}
$$

where

$k_{22}=(1-\eta) k_{2}+\eta k_{1} ; \quad B_{2}=\frac{(1-\eta) k_{2} b_{2}+\eta k_{1} b_{1}}{(1-\eta) k_{2}+\eta k_{1}}$. Therefore, $\mathcal{K}_{22}$ verifies Klinkenberg's law whereas $\mathcal{K}_{11}$ doesn't, which shows that for the flow in a bilaminated composite, Klinkenberg's law survives upscaling for a flow parallel to the layers but it doesn't for a flow perpendicular to the layers.

\section{ACOUSTICS OF GAS AT LOW PRESSURE IN A POROUS MEDIUM}

\subsection{Medium description}

In this section we consider a periodic rigid porous medium saturated by a gas at low pressure and which is submitted to a monochromatic excitation of 
wave-length $L$. We assume that the gas pressure is sufficiently low to give rise to a wall-slip effect at the pore scale. The goal of this work consists in applying homogenisation to the description at the pore scalc that consists of the equations of linear acoustics as in (Levy 1979) and (Auriault 1980), but with the wallslip condition on the solid-liquid interface which is used in (Skjetne \& Auriault 1999) in the case of quasistatics. The period which corresponds to the pore scale is denoted by $\Omega$ and is of characteristic length $l \ll L$. Within the period, $\Omega_{p}$ and $\Omega_{s}$ represent the domains occupied by the pore space and by the solid, respectively, and $\Gamma$ denotes their common boundary, In harmonic regime, we thus consider the following local description:

In $\Omega_{p}$ :

$\mu \nabla^{2} \boldsymbol{v}+(\eta+\mu) \nabla(\nabla \cdot v)-\nabla_{p}=i \omega \rho^{e} \boldsymbol{v}$,

$i \rho \omega+\rho^{e} \nabla \cdot(v)=0$,

$p=A \rho$,

On $\Gamma$;

$v=-c \lambda t_{1} \cdot(\nabla v) \cdot n t_{1}$,

where $\mu$ and $\eta$ are the viscosities, $\rho^{e}$ is the density at the equilibrium, $c$ is a constant and $\lambda$ is the mean free path. Vectors $\boldsymbol{t}_{1}$ and $\boldsymbol{n}$ represent the unit tangential and the unit normal to $\mathrm{r}$, respectively.

Let now normalise the above local description: it consists in defining the set of dimensionless numbers that characterize the local description and then in estimating them with respect to the scale ratio $\varepsilon=I / L$. From the above local description we define the four following dimensionless numbers:

$$
\begin{aligned}
& Q=\frac{|\nabla p|}{\left|\mu \nabla^{2} v\right|} ; \quad R_{f}=\frac{\left|i \omega \rho^{e} v\right|}{\left|\mu \nabla^{2} v\right|} ; \\
& S=\frac{|i \rho \omega|}{\left|\rho^{e} \nabla \cdot v\right|} ; \quad K_{n}=\frac{\left|-c \lambda d_{1} \cdot(\nabla v) \cdot \boldsymbol{m} t_{1}\right|}{|v|} .
\end{aligned}
$$

In order to estimate these dimensionless numbers, let consider $l$ as the reference length. The dimensionless number $Q$ is the ratio of the pressure gradient to the viscous forces. It can be shown (Auriault 1991) that $Q_{1}=O\left(\varepsilon^{-1}\right)$. The transient Reynolds number, $R_{i}$, is the ratio of the transient term to the viscous term. Under acoustic perturbations the inertial term does act at the local scale, which reads $R_{t}=O(1)$. The Strouhal number, $S$, characterizes the transient behaviour of flow and a macroscopic transient behaviour corresponds to $S_{l}=O(1)$. The Knudsen number $K_{n}$ is a measure of a the wall-slip effect. By definition, it is also written as:

$K_{n}=\frac{\mu \sqrt{\pi R T / 2}}{l p^{e}}$,

Let us assume, in the first place, that $K_{n}=O(1)$.
Thus, we obtain the following dimensionless local description:

$$
\begin{aligned}
& \nabla^{2} \boldsymbol{v}+\nabla(\nabla \cdot v)-\varepsilon^{-1} \nabla p=i \omega \rho^{e} \boldsymbol{v} \text { in } \Omega_{p}, \\
& i \omega \varepsilon \rho+\rho^{e} \nabla \cdot \boldsymbol{v}=0 \quad \text { in } \Omega_{p}, \\
& p=A_{\rho} \quad \text { in } \Omega_{p}, \\
& v=-K_{n} \boldsymbol{t}_{1} \cdot(\nabla v) \cdot n t_{1} \quad \text { on } \mathrm{I} .
\end{aligned}
$$

\subsection{Homogenisation}

The procedure in the same as in 2.2 i) the physical variables (pressure, velocity and density) are looked for in the form of asymptotic expansions in powers of $\varepsilon$ and these expansions are incorporated in the local description (32)-(35); ii) the boundary-value problems that arise at the successive orders of $\varepsilon$ are solved.

\subsubsection{First-order pressure, density and velocity fields}

From equations (32) and (34) at the first order, we deduce:

$p^{(0)}=p^{(0)}(\boldsymbol{x}, t)$ and $\rho^{(0)}=\rho^{(0)}(\boldsymbol{x}, t)$.

The second-order problem reads:

$$
\begin{aligned}
& \nabla_{y}^{2} v^{(\theta)}-\nabla_{x} p^{(0)}-\nabla_{y} p^{(1)}=i \omega \rho^{\prime} v^{(\theta)} \quad \text { in } \Omega_{p}, \\
& \nabla_{y} \cdot v^{(\theta)}=0 \text { in } \Omega_{p}, \\
& v^{(\theta)}=-K_{n} t_{1}^{(\theta)} \cdot \nabla_{y} v^{(\theta)} \cdot n t_{1}^{(0)} \quad \text { on } \Gamma .
\end{aligned}
$$

The solution to this system may be written as follows: $\boldsymbol{v}^{(\boldsymbol{0})}=-\boldsymbol{k}\left(\boldsymbol{y}, \boldsymbol{K}_{n}, \omega\right) \boldsymbol{\nabla}_{x} p^{(0)}$.

Its average over the period yields:

$$
\left\langle v^{(0)}\right\rangle=-\boldsymbol{K}\left(K_{n}, \omega\right) \nabla_{x} p^{(0)},
$$

where the effective dynamic permeability tensor $\boldsymbol{K}$ is a complex-valued and depends upon the pulsation and the Knudsen number.

\subsubsection{Small Knudsen number}

In order to get more insight into the expression of $\boldsymbol{K}$. we consider the case of low Knudsen number:

$\varepsilon \ll K_{n} \ll 1$.

As in (Skjetne \& A uriault 1999), $v^{(0)}$ and $p^{(1)}$ are looked for in the form of asymptotic expansions of $K_{n}$ :

$$
\begin{aligned}
& v^{(0)}=v^{0}+K_{n} v^{1}+\left(K_{n}\right)^{2} v^{2}+\cdots \\
& p^{(1)}=p^{0}+K_{n} p^{1}+\left(K_{n}\right)^{2} p^{2}+\cdots
\end{aligned}
$$


Substituting the above expansions into boundaryvaluc problem $(36)-(38)$ yields at the first order:

$\nabla_{y}^{2} v^{(\theta)}-\nabla_{x} p^{(0)}-\nabla_{y} p^{0}=i \omega \rho^{e} v^{\theta} \quad$ in $\Omega_{p}$,

$\nabla_{y} \cdot v^{0}=0$ in $\Omega_{p}$,

$v^{0}=0$ on $\Gamma$.

The solution to this linear well-posed problem is:

$v^{0}=-\boldsymbol{K}^{0}(\boldsymbol{y}, \omega) \nabla p^{(0)}$,

and its average over the period is written as:

$\left\langle v^{0}\right\rangle=-\boldsymbol{K}^{0}(\omega) \nabla p^{(0)}$,

where

$K^{0}=\frac{1}{|\Omega|} \int_{\Omega p} k^{0} d \Omega$

The second rank complex permeability tensor $\boldsymbol{K}^{\mathbf{0}}$ is symmetrical and positive (Sanchez-Palencia 1980).

At the second order, we obtain the following wallslip correction problem:

$\nabla_{y}^{2} v^{1}-\nabla_{y} p^{1}=i \omega p^{\prime} v^{1}$ in $\Omega_{p}$,

$\nabla_{y} \cdot v^{1}=0 \quad$ in $\Omega_{p}$,

$v^{1}=-t_{1}^{(\theta)} \cdot \nabla_{y} v^{0} \cdot n t_{1}^{(0)}$ on $\mathrm{r}$,

The average over the period of the solution $v^{\prime}$ to the above well-posed problem is written as:

$\left(v^{1}\right)=-\boldsymbol{K}^{1}(\omega) \nabla p^{(0)}$,

in which $\boldsymbol{K}^{1}$ is a complex permeability tensor.

According to (46) and (51), the average of expansion (41) up to the second order leads to:

$\left(v^{(0)}\right)=-\left(\boldsymbol{K}^{0}+K_{n} \boldsymbol{K}^{\mathbf{1}}\right) \nabla p^{(0)}$,

which can also be written as:

$\left(v^{(0)}\right)=-\boldsymbol{K}^{0}(\omega)\left(1+K_{n} \boldsymbol{H}(\omega)\right) \boldsymbol{\nabla} p^{(0)}$.

Considering the definition of the Knudsen number (31), it appears that the structure of Equation (53) seems to be similar to Klinkenberg's law (1), but with the difference that constants $k_{\infty}$ and $c$ have been replaced by the pulsation dependent tensors $\boldsymbol{K}^{0}$ and $\boldsymbol{H}$, respectively.

When taking $\omega=0$, the inertial terms in systems $(43)-(45)$ and $(48)-(50)$ vanish. It corresponds to the quasi-statics case investigated in (Skjetne \& Auriault 1999).

Written in time space, Equation (53) would include a convolution product which characterizes memory effects. The presence of these effects is a well-known result in the classical case of acoustics in porous media (Auriault 1980) and, therefore, they are not due to the wall-slip flow. In order to illustrate the above results, and to examine the influence of the wall-slip effect upon the effective tensors $\boldsymbol{K}^{\mathbf{0}}$ and $\boldsymbol{H}$, we now consider the problem on the simple geometry of a bundle of capillaries.

\subsection{Illustration on a simple geometry: bundle of capillary tubes}

The purpose of this section is to examine the macroscopic law for the acoustics with wall-slip effects of a bundle of capillaries of radius $a$ saturated by a gas (Figure 2). According to the results obtained in the previous section, the macroscopic behaviour is described by

$\left\langle v^{(0)}\right\rangle=-K_{\mathrm{eff}}(\omega) \frac{d p^{(0)}}{d x}$.

The dynamic permeability $K_{\text {eff }}$ is defined by

$K_{\mathrm{cII}}=\frac{\phi}{\pi a^{2}} \int_{0}^{\hat{a} 2 \pi r k(r, \omega) d r,}$

where $k$ is such that $v^{(0)}=-k \frac{d y^{(0)}}{d x}$ is the solution to the local boundary-value problem (36)-(38), which in a single capillary tube reduces to:

$\frac{d^{2} v^{(0)}}{d r^{2}}+\frac{1}{r} \frac{d v^{(0)}}{d r}-\frac{i \omega \rho^{e}}{\mu} v^{(0)}=\frac{1}{\mu} \frac{d p^{(0)}}{d x}$.

$\frac{1}{\mu} \frac{d p^{(1)}}{d r}=0$

$v^{(0)}(a)=-c \lambda \frac{d v^{(0)}}{d r}$.

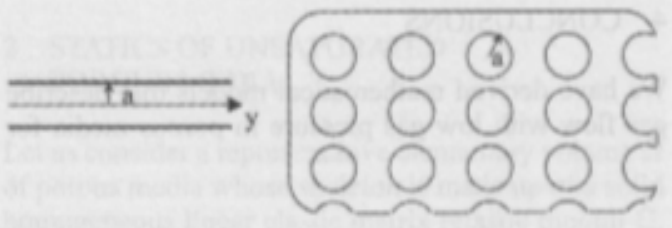

(a)

(b)

Figure 2. Bundle of cylindrical capillaries: (a) a single capillary $\boldsymbol{y}$-variable; (b) Medium cross-section in $\boldsymbol{x}$-variable. 


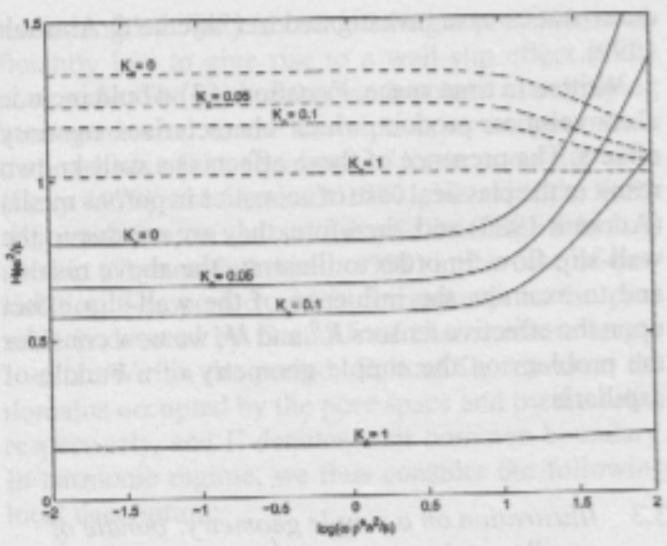

Figure 3. Variations of $H_{1}$ and $I_{2}$ with respect to the dimensioless pulsation. Plain line: $0.1 H_{1}$ (dissipative term); Dashed line: $\omega^{-1} H_{2}$ (inertial term).

Since there is an analytical solution to the above boundary-value problem, it can easily be deduced that:

$K_{\mathrm{eff}}=-\frac{\phi}{i \omega \rho^{e}} \frac{J_{2}(\alpha a)+K_{n} a \alpha J_{1}(\alpha a)}{J_{0}(\alpha a)-K_{n} a \alpha \cdot J_{1}(\alpha a)}$,

where $J_{i}$ are Bessel functions and $\alpha=i \sqrt{i \omega \rho^{2} / \mu}$.

It can be secn from Equation (58) that Klinkenberg's law (1) is not verified.

Let us define the inverse permeability by $K_{\text {eff }}^{-1}=$ $H_{e}=H_{1}+i H_{2}$. The term $H_{1}$ represents the dissipation whereas $\mathrm{H}_{2} \omega^{-1}$ is the inertial term. The curves of $H_{1}$ and $H_{2} \omega^{-1}$ with respect to the dimensionless pulsation $\omega^{*}=\omega \rho^{e} \alpha^{2} / \mu$ are plotted on figure 3 for different values of $K_{n}$. When $K_{n}=0$ the profiles of both curves are those obtained in the case of acoustics without Klinkenberg's effect: the inertial term tends towards 1 whereas the dissipation term increases with the pulsation. The enhanced value of $H_{1}$ (i.e. greater than 1) at low pulsation is due to the added mass effect. When Knudsen number is increased, both the dissipative and the inertial term decrease as a result of the increase of the wall-slip effect.

\section{CONCLUSIONS}

We have derived mathematical models that describe gas flow with low gas pressure in porous media for two distinct problems: i) the flow of gas at low pressure in a composite porous media; ii) acoustics with wallslip effect of a porous medium saturated by a gas. In both problems we have shown that in the most general cases Klinkenberg's law is not verified at the macroscopic scale. In the first problem, we have seen that Klinkenberg's law may be verified on specific geometries. In the second problem, it is shown that the macroscopic behaviour is slightly modified when a wall-slip effect is considered at the pore scale: both the added mass effect and the energy dissipation are reduced.

\section{REFERENCES}

Auriault, J.-L. 1980. Dynamic behaviour of a porous medium saturated by a newtonian fluid. Int. J. Eng. Sc. 18 : 775-785.

Auriault, J.-L. 1983. Effective macroscopic description for heat conduction in periodie composites. Int. J. Heat Mass Transfer 26(6): 861-869.

Auriault, J.-L. 1991. Heterogeneous medium: is an equivalent macroscopic description possible? Int. J. Engng Sci. 29, n7: $785-795$.

Baehr, A.L. \& M.F. Hult 1991. Evaluation of the unsaturated zone air permeability through pneumatic tests. Water Resourc. Res. 27 (10): 2605-2617.

Benssoussan, A., J.-L. Lions, \& G. Papanicolaou 1978. Asymptotic Analysis for Periodic Sinuctures. Amsterdam North-Holland Publishing Company.

Fun-Gau-Ilo \& W. Strieder 1982. A mean free path theory of void diffusion in a porous medium with surface diffusion. II: Numerical evaluation of the effective diffusivity for arbitrary knudsen number. Journal of Chemical Physics 76 , no. 1: 673-677.

Jones, S.-C. 1972. A rapid accurate unsteady-state klinkenberg permeameter. Petrol. Engrs.: 383-397.

Klinkenberg. L.-J. 1941. The permeability of porous media to liquids and gas. Drilling and Production Practice, American Petroleum Inst:: 200-213.

Levy, T. 1979. Propagation of waves in a fluid saturated porous elastic solid. Int. J. Engn. Sci. 17: 10051014.

Sanchez-Palencia, E. 1980. Non-homogeneous media and vibration theory. Lecture Notes in Physics, SpringerVerlag Berlin 127.

Skjetne, E. \& J.-L.. Auriault 1999. Ilomogenization of wallslip gas flow through porous media. Trunsport in Pomes Media 36: 293-306.

Skjetne, E. \& J.S. Gudmundsson 1993. Model for wall-slip in the darey and forchheimer gas flow regimes. Norwegian Institut of Technology, pp. 111-122. Tapir Publishers.

Wu, Y.-S. K. Pruess, \& P. Persoff 1998. Gas flow in porous media with klinkenberg effects. Transport in Porows Media 32: 117-137. 